\title{
3D Metamaterials with Negative Thermal Expansion and Negative Effective Compressibility
}

\author{
Jingyuan Qu, Muamer Kadic, Andreas Naber, Alexander Gerber, \\ Frederik Mayer, and Martin Wegener
}

\begin{abstract}
Materials with negative thermal expansion are desired for controlling thermal stresses, but unusual in nature. With two-component metamaterials it is possible to tune the thermal expansion from positive over zero to negative values, even if both components have positive thermal expansion. Using gray-tone laser lithography we fabricate three-dimensional two-component polymer microlattices, exhibiting zero or negative thermal expansion [1].

Similarly, poroelastic metamaterials can have a negative effective compressibility [2]. By using the high compressibility of air or vacuum, only one constituent solid component is needed here. At an elevated pressure, the effective metamaterial volume increases, while the volume defined by the constituent solid still decreases. Thus the metamaterial with this counterintuitive property is still stable.

The thermal expansion and effective compressibility of fabricated samples are measured by cross-correlating optical images taken at different temperatures or pressure respectively. The results ar in good agreement with numerical calculations.
\end{abstract}

\section{References}

1. Qu J, Kadic M, Naber A, Wegener M (2017) Sci Rep 7:40643

2. Qu J, Kadic M, Wegener M (2017) Appl Phys Lett 110:171901

J. Qu (ه) $\cdot$ F. Mayer $\cdot$ M. Wegener

Institute of Applied Physics, Karlsruhe Institute of Technology (KIT), Karlsruhe, Germany Institute of Nanotechnology, Karlsruhe Institute of Technology (KIT), Karlsruhe, Germany e-mail: jingyuan.qu@kit.edu

M. Kadic

Institute of Applied Physics, Karlsruhe Institute of Technology (KIT), Karlsruhe, Germany Institut FEMTO-ST, CNRS, Université de Bourgogne Franche-Comté, Besançon, France

A. Naber · A. Gerber

Institute of Applied Physics, Karlsruhe Institute of Technology (KIT), Karlsruhe, Germany

(C) Springer Nature B.V. 2018

B. Di Bartolo et al. (eds.), Quantum Nano-Photonics, NATO Science for Peace

and Security Series B: Physics and Biophysics,

https://doi.org/10.1007/978-94-024-1544-5_47 